\title{
Interdisciplinary collaboration in a case of scrotal extramammary Paget's disease
}

\author{
Éva Anna Piros ${ }^{1,2}$, Judit Ihász ${ }^{2}$, Péter Riesz ${ }^{3}$ \\ ${ }^{1}$ Department of Dermatology, Venerology, and Dermato-oncology, Semmelweis University, Budapest, Hungary \\ 2Private Clinic of Dermatology, Budapest, Hungary \\ ${ }^{3}$ Department of Urology, Semmelweis University, Budapest, Hungary
}

Adv Dermatol Allergol 2021; XXXVIII (6): 1105-1107 DOI: https://doi.org/10.5114/ada.2020.94924

Extramammary Paget's disease (EMPD) is an infrequent tumour that is characterised by a chronic eczemalike rash around the anogenital region. The most common symptom of extramammary Paget's disease is mild or intense itching around the lumbar, genital, perineal, or perianal regions. Pain and bleeding may result from potential injuries. The thick plaques can be red, squamous, and rough. The first symptoms are itching and burning from one or more persistent plaques. Due to the rarity of the disease, it is difficult to find a specialist. Differential diagnosis is complicated by the fact that EMPD symptoms can mimic those of other diseases [1]. The diagnostic process should include physical, microscopic, immunohistochemical, and interdisciplinary examinations. EMPD is usually treated surgically by removing the skin lesion [2-4]. In some cases, treatment can be effective, such as photodynamic and imiquimod treatment, radiotherapy, chemotherapy, androgen therapy, and $\mathrm{CO}_{2}$ laser ablation [5-7].

Male patients use the term "jock itch" for the symptoms. In some cases, there is mild sensitivity or there are no symptoms in the affected region. The disease most often occurs between the ages of 50 and 70 years, but it can also appear earlier [8]. The disease is infrequent, and usually the progress is slow. If the disease is diagnosed early, the prognosis is good [9]. EMPD can also be aggressive and invade the dermis and subcutaneous tissues. As a consequence, invasive carcinoma and metastases can develop. This is the reason why physical examinations are very important in the following regions: axillary, genital, and anal [10]. The most commonly affected areas among women are the vulva or the anus, but the disease can affect the mons pubis, vagina, and thighs. The most commonly affected areas among men are the genital organs (scrotum, penis), anal, perianal, lumbar regions, and thighs. Tumours that invade the eyelids, scalp, nose, and acoustic meatus are extremely rare. EMPD is located on the apocrine gland-rich areas: axilla, inguinal area, scrotum, vulva, retroauricular region, and sweat glands [11]. For surgical treatment, it is important to determine the extension of the tumour [12]. When EMPD is suspected, the diagnosis is typically confirmed via a skin biopsy of the lesion. The location of extramammary Paget's disease is useful in predicting the risk of associated cancer. In some cases, EMPD appears 10-15 years before invasive carcinoma and metastases. Approximately $25-35 \%$ of EMPD cases around the anal region are associated with underlying colorectal cancer. The mortality of the disease is low. The prognosis depends on the association with an underlying cancer.

A 62-year-old man had had an inflamed and eczemalike lesion in the right inguinal and scrotal regions since 2011. He had visited several dermatologists for 6 years. Antimicrobial, antifungal, and local treatments were not successful. In 2015, the disease invaded the left scrotal region. Because of the disease's long-standing nature and resistance to treatment, a skin biopsy was performed in September 2017. The biopsy result was extramammary Paget's disease. The laboratory, medical, urological, and radiological (chest X-ray, abdominal US, abdominal + pelvic MR/CT) examinations were negative. An uro-oncology and radiotherapy consultation suggested irradiation because of the tumour's wide extension and its locations (Figures $1 \mathrm{~A}-\mathrm{C}$ ). Between October 30 and November 30, 2017 the patient received $20 \times 2$ Gy half-deep X-ray irradiations [13].

Macroscopic description: the histological sample was a $10 \times 3 \mathrm{~mm}$ part of the skin with a $5 \mathrm{~mm}$ brownish and off-white papule on the surface.

Microscopic description: the biopsy was taken from the lumbar lesion that had existed for 6 years. The surface of the biopsy was hyperkeratotic and covered by slightly

Address for correspondence: Éva Anna Piros MD, Department of Dermatology, Venerology and Dermato-oncology, Semmelweis University, Mária u. 41, 1085 Budapest, Hungary, phone: +36 306183568, fax: +36 12305002, e-mail: piros.anna95@gmail.com

Received: 10.02.2020, accepted: 1.04.2020. 

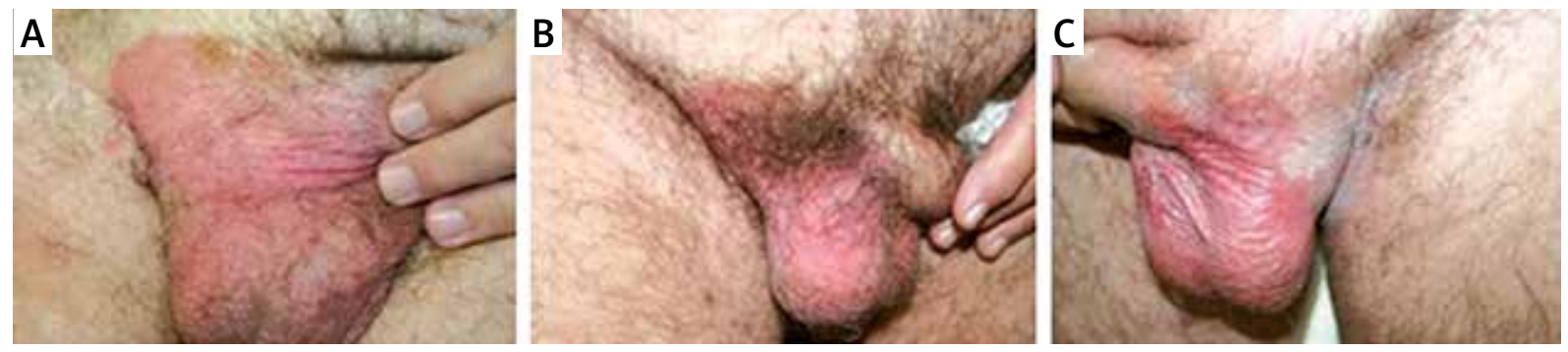

Figure 1. A-C - Extramammary Paget's disease (EMPD) at scrotum
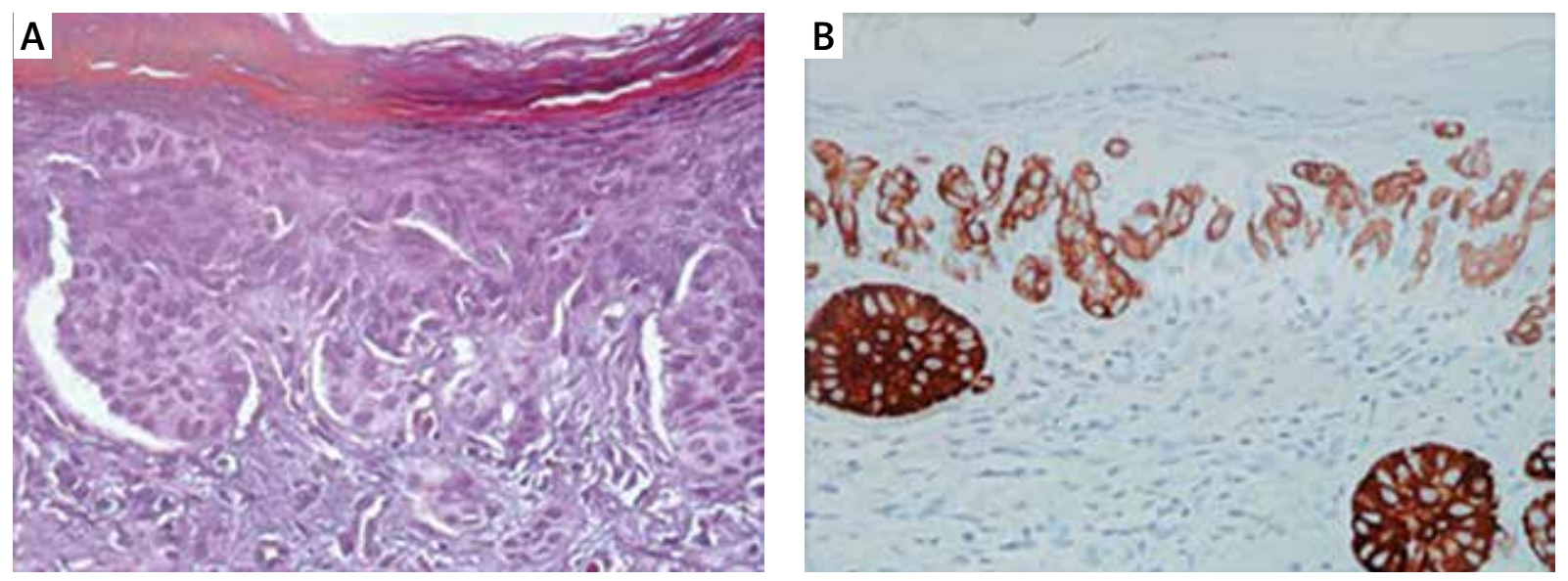

Figure 2. Histopathologic examination. The thickness of the sample is $2 \mu \mathrm{m}$. A - Haematoxylin-eosin (HE) staining. B - Immunohistochemical (IH) reaction with cytokeratin-7 (CK7) positivity (CK+)

expanded, multilayer keratinised epithelium [14, 15]. At the basal part of the epithelium, atypical cells with wide, pale cytoplasm were recognised in variable-sized nests (Figures 2 A, B). The nuclei were large and variable-sized with a loose chromatin structure and prominent nucleoli. Similar cells in smaller nests or individually infiltrating the full thickness of the epithelium were observed, and in some parts, the granular and keratinised layers were also affected. Similar cells either individually or in small groups were recognised in the epithelium of the skin tag, dermis, and deeper part of the reticular dermis. The lesion reached the edge of the biopsy excision. The immunohistochemical examination showed cytokeratin positivity and partial positivity for cytokeratin-7 protein. Regarding cytokeratin-20, the sample was negative $[11,15]$. The sample was negative for oestrogen receptor, PSA, and S-100 protein.

Diagnosis: extramammary Paget's disease with dermal infiltration.

After completing radiotherapy, we followed up the patient every 3 months. After 1 year, in November 2018, all the above-mentioned examinations were performed again. The examinations did not show any tumour recurrences or metastases.

When chronic inflammation exists for several years, we should consider systemic diseases and malignant lesions. In many cases, histological examinations are need- ed for the correct diagnosis. EMPD is a complex disease, so interdisciplinary collaboration with several doctors is needed for a cure. Depending on the location of the disease, the therapist can be a dermatologist, general surgeon, plastic surgeon, colorectal surgeon, oncologist, urologist, or gynaecologist.

\section{Conflict of interest}

The authors declare no conflict of interest.

\section{References}

1. Lam C, Funaro D. Extramammary Paget's disease: summary of current knowledge. Dermatol Clin 2010; 28: 807-26.

2. Yang WJ, Kim DS, Im YJ, et al. Extramammary Paget's disease of penis and scrotum. Urology 2005; 65: 972-5.

3. Mengjun B, Zheng-Qiang W, Tasleem MM. Extramammary Paget's disease of the perianal region: a review of the literature emphasizing management. Dermatol Surg 2013; 39: 69-75.

4. Meng F, Pu Y, Chen Z. Comparison of wide local excision and radical excision for Paget's disease involving the penis and scrotum. Zhongguo Xiu Fu Chong Jian Wai Ke Za Zhi 2017; 31: 714-7.

5. Dos Santos JS, Bonafé GA, Pereira JA, et al. Rare perianal extramammary Paget disease successfully treated using topical Imiquimod therapy. BMC Cancer 2018; 18: 921. 
6. Kim TH, Chang IH, Kim TH, et al. Extramammary Paget's disease of scrotum treated with radiotherapy. Urology 2009; 74: 474.e1-3.

7. Gray J, Gray A, Swan J, et al. Perianal extramammary Paget disease treated with topical imiquimod and oral cimetidine. Cutis 2018; 101: E19-22.

8. Lopes Filho LL, Lopes IM, Lopes LR, et al. Mammary and extramammary Paget's disease. An Bras Dermatol 2015; 90 : 225-31.

9. Maeda T, Yoshino K, Nagai K, et al. Successful outcome of early ambulation after extensive skin grafting in extramammary Paget's disease. J Dermatol 2018; 45: 1456-8.

10. Shepherd V, Davidson EJ, Davies-Humphreys J. Extramammary Paget's disease. BJOG 2005; 112: 273-9.

11. Isrow D, Oregel KZ, Cortes J, et al. Advanced extramammary Paget's disease of the groin, penis, and scrotum. Clin Med Insights Oncol 2014; 8: 87-90.

12. Kato N, Matsue K, Sotodate A, et al. Extramammary Paget's disease with distant skin metastasis. J Dermatol 1996; 23: 408-14.

13. Li YC, Lu LY, Yang YT, et al. Extramammary Paget's disease of the scrotum associated with hepatocellular carcinoma. J Chin Med Assoc 2009; 72: 542-6.

14. Ekwueme KC, Zakhour HD, Parr NJ. Extramammary Paget's disease of the penis: a case report and review of the literature. J Med Case Reports 2009; 3: 4.

15. Nakamura Y, Fujisawa Y, Ishikawa M, et al. Usefulness of sentinel lymph node biopsy for extramammary Paget's disease. Br J Dermatol 2012; 167: 954-6. 